Splenic angiosarcoma has been presented as an extremely rare malignant tumour. Amongst the rarest of all neoplasms, primary splenic angiosarcoma comprises $0.14-0.25$ of all annually reported cases per one million persons Splenic angiosarcoma was first described in 1879 by T. Langerhans. The clinical symptoms and diagnostic values associated with splenic angiosarcoma are extremely variable. Nonetheless, majority of the patients (75\%) complain of abdominal pain, and a quarter to onethird present with rupture of the involved organ. We hereby report our experience with two splenic angiosarcoma cases, which despite being similar in their diagnosis, nonetheless present with an entirely different clinical picture. Our first case, whereby the patient presented with a liver that was also affected by the angiosarcoma of the spleen. In the second case however, although the patient did show evidence of metastasis to the abdominal cavity as well as the liver, she also suffered from primary adenocarcinoma of the colon. To our knowledge, no previous article has made mention of primary splenic angiosarcoma existing independently of another primary neoplasm. Both may be found in common practice, and ought to be highlighted for their significance as such.

Key words: primary splenic angiosarco ma, primary colon cancer, splenectomy.

\section{Primary splenic angiosarcoma - the same diagnosis yielding two different clinical pictures. Case report}

\author{
Zbigniew Kamocki', Agnieszka Steward, Konrad Piotr Zaręba², \\ Adam Kukliński, Boguław Kędra²
}

1Department of Medical Pathomorphology, Medical University of Bialystok, Poland $22^{\text {nd }}$ Department of General and Gastroenterological Surgery, Medical University of Bialystok, Poland

\section{Introduction}

Although splenic angiosarcoma has been presented as an extremely rare malignant tumour in medical literature spanning the last 20 years, an increasing number of cases have been identified over that same time period. Some of these cases are typically identified upon spontaneous rupture of the organ $(\sim 32 \%)$, and as such are associated with a poor prognostic outcome for the patient. Despite tremendous strides undertaken in medicine over the last two decades, both conservative and invasive treatment measures continue to produce low-yield results when it comes to prolonging patient survival. There is no evidence to suggest a clinical benefit of adjuvant chemotherapy [1]. This may likely be attributed to a high rate of distant metastasis, affecting organs such as the liver, breast and bone. Only Hara et al. have reported long-term survival of a patient with angiosarcoma after splenectomy, and high-dose chemotherapy, and autologous peripheral blood stem cell transplantation [2]. We hereby report our experience with two splenic angiosarcoma cases, which despite being similar in their diagnosis, nonetheless presented with an entirely different clinical picture. Both may be found in common practice, and ought to be highlighted for their significance.

\section{Case 1}

A 54-year-old Caucasian man was initially admitted to the Clinic of Gastroenterology with non-specific complaints of abdominal pain and loss of appetite over the last 6 weeks, which grew worse post-prodigally and with respiration. Laboratory results revealed normocytic anaemia (haemoglobin $10.0 \mathrm{~g} / \mathrm{dl}$ ), reticulocytosis, thrombocytopenia (platelets $\left.68 \times 10^{9} / \mathrm{l}\right)$, a small increase in bilirubin (2.34 mg/dl), as well as elevated LDH (758 IU), GGT (200 IU) and ALP (336 IU) levels. Ultrasound was inconclusive, but revealed structural changes that corresponded to either the presence of metastasis or a haemangioma. A subsequent CT scan further elucidated an affected spleen, with possible metastatic or lymphoma-like changes (Fig. 1).

The patient was scheduled for explorative surgery, but prior to it being performed, he went into shock and presented with an ascitic abdomen. His subsequent morphological parameters were increasingly depressed when compared to their previous counterparts, and emergency ultrasound and CT scans showed a significant amount of peritoneal fluid. During the emergency surgery, approximately 3 litres of both old and fresh blood were discovered within the peritoneal cavity, along with a $16 \mathrm{~cm}$ cyst located in the spleen, which was also seeping blood. Both lobes of the liver were covered with numerous small tumours, 2 of which were bleeding, as they sat interspersed between multiple blood clots. After suturing and a histological sample of the liver had been 


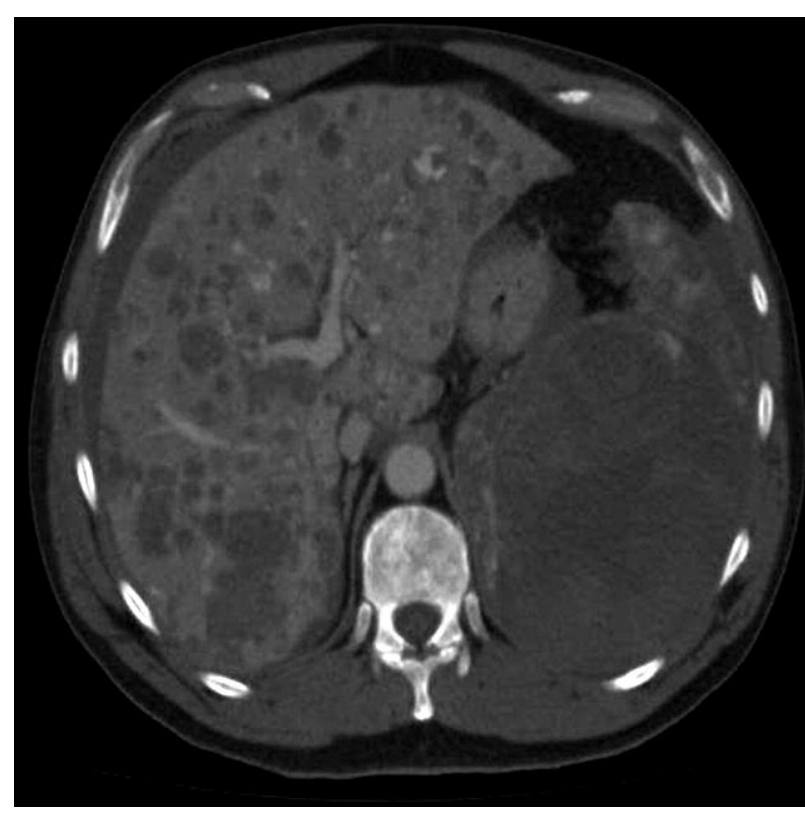

Fig. 1. Abdominal computed tomography scan demonstrating an enlarged spleen with the metastatic liver tumours

obtained, a splenectomy and peritoneal lavage with warm saline to evacuate the clots were performed, and the operation was concluded. A cross-section of the spleen subsequently revealed a greyish-white discharge, with the organ itself measuring $16 \times 12 \times 10 \mathrm{~cm}$ (Fig. 2). Post-operatively, the patient presented with no complications and was subsequently released. He died 3 months after his operation.

\section{Case 2}

A 77-year-old Caucasian woman was admitted to our hospital due to haematochezia and fatigue. She initially presented at the clinic with a number of concurrent illnesses, chief amongst them being normocytic anaemia, diabetes mellitus type 2, hypertension, and paroxysmal atrial fibrillation. Cancer of the sigmoid colon was detected by colonoscopy. Having previously undergone a hysterectomy, the patient had now been referred for the operation. The spleen was visibly enlarged, and showed a number of superficial tumours. The liver was also bilaterally distended, without tumours. Purpura-like changes were further noted atop the greater omentum, as well as the wall and mesentery of the small

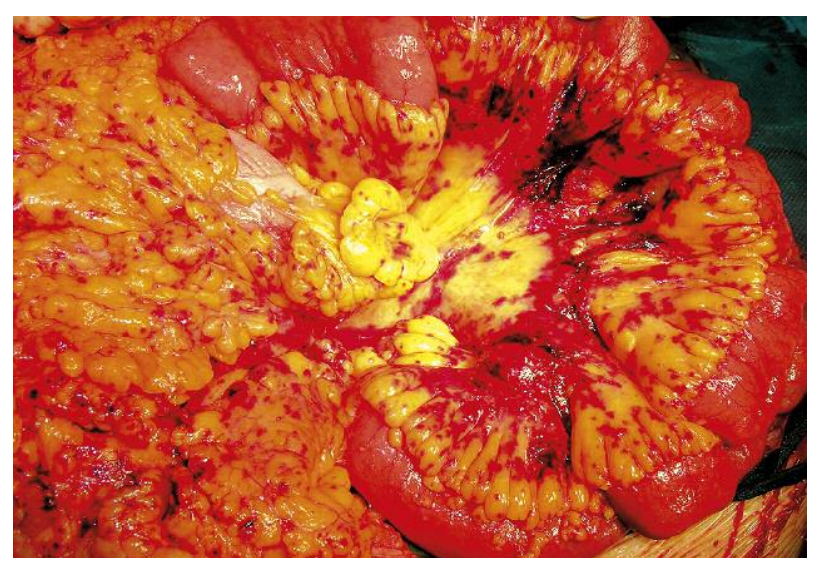

Fig. 3. Intraperitoneal dissemination of the splenic angiosarcoma

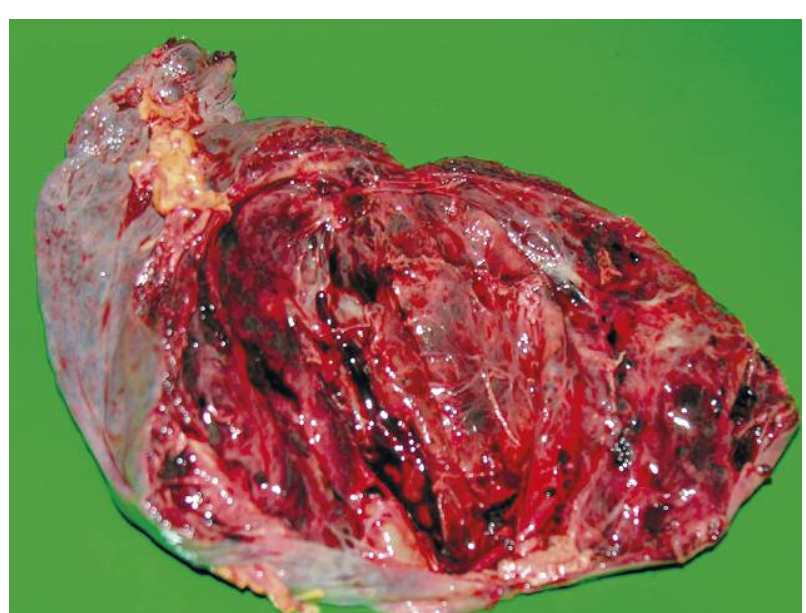

Fig. 2. The excised spleen with haemorrhagic and nodular lesions

bowel (Fig. 3). At the sigmoid portion of the colon, immediately above the colorectal junction, a $2 \mathrm{~cm}$ tumour was observed (Fig. 4). A resection of the sigmoid colon as well as the anterior portion of the rectum was undertaken, with an accompanying colorectal anastomosis. The regional lymph nodes were also removed, as was the spleen (Fig. 5). Subsequent histological examination of the obtained samples evaluated them to be an adenocarcinoma of the sigmoid colon (G2, T3), an angiosarcoma of the spleen (G3), as well as lymph nodes that were affected by metastasis (Fig. 6). No complications were noted following the surgery, although the patient's state was deemed to be less than ideal. Her age and the concurrent presentation of two independent cancers excluded her from the possibility of receiving adjuvant chemotherapy. She died approximately a month later.

\section{Discussion}

Amongst the rarest of all neoplasms, primary splenic angiosarcoma has a frequency of 0.14-0.25 reported cases

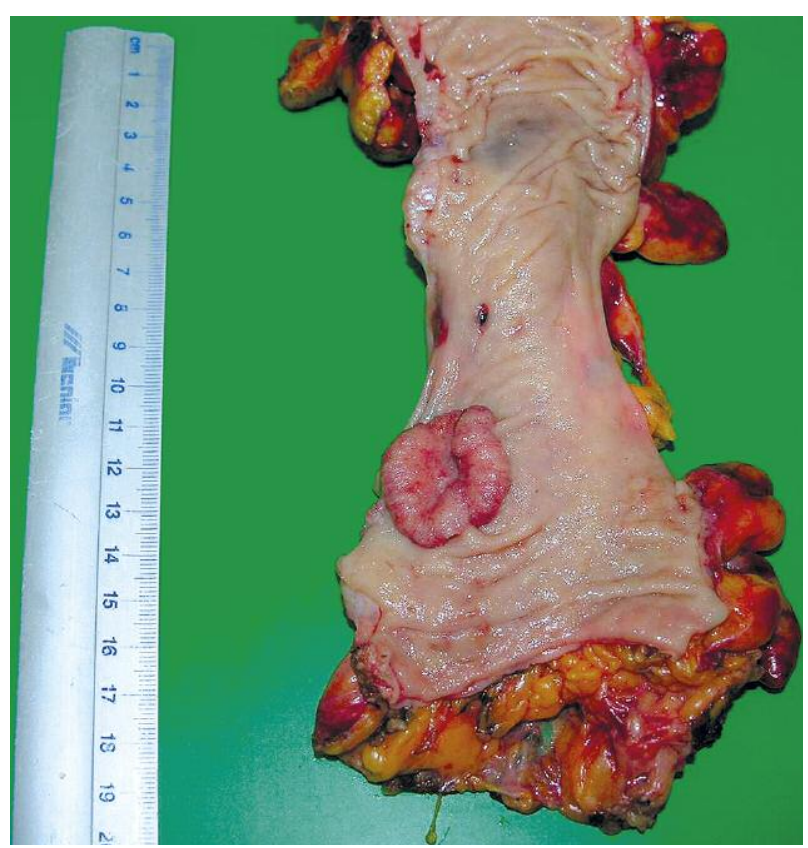

Fig. 4. Carcinoma of the sigmoid colon 


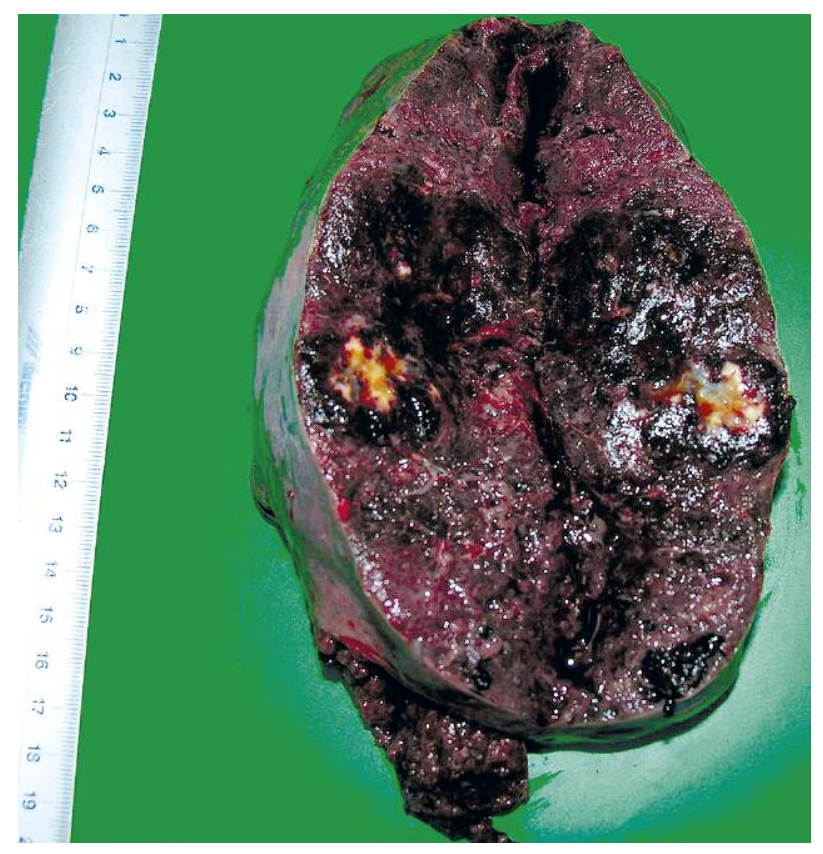

Fig. 5. The splenic angiosarcoma

per one million persons annually [3, 4]. Splenic angiosarcoma was first described in 1879 by T. Langhans [5]. To our knowledge, no previous article has made mention of primary splenic angiosarcoma existing independently of another primary neoplasm. Although Jiménez-Heffernan et al. (1999) have outlined the rupture of a splenic angiosarcoma into the colon - subsequently presenting as anal bleeding - they made no mention of the event occurring as a result of synchronous presentation of two primary tumours [6]. More accurately, these results suggest that the affected splenic flexure was a site of a Gl metastasis, rather than primary neoplastic involvement. As such, our case would be the first one to highlight the co-occurrence of a simultaneous adenocarcinoma of the colon and an angiosarcoma of the spleen.

The clinical symptoms and diagnostic values associated with splenic angiosarcoma are extremely variable. Nonetheless, the majority of patients (75\%) complain of abdominal pain, and a quarter to one-third present with rupture of the involved organ [3-12]. Anaemia, leucopoenia, elevated LDH levels and thrombocytopenia are also frequently reported, with anaemia being caused by damage to the red cell surface, whilst the other factors may be precipitated by severe haemolysis [8]. It has been increasingly postulated that thrombocytopenia may be the first manifestation of the disease, even in the absence of other laboratory irregularities [10]. Interestingly, while both cases are characterized by similarities when it comes to the values obtained in their laboratory results, the first patient had never suffered from any major illness prior to his presentation of splenic angiosarcoma, while the second had long been affected by a number of other illnesses.

Splenic angiosarcoma typically carries with it a grave prognosis for all but $20 \%$ of patients, who then manage to survive for a period longer than 6 months $[4,6]$. Survival statistics are thought to be increased in those instances where the diagnosis and/or the splenectomy are performed prior to the actual rupture of the organ $[3,11]$. The patients dis-

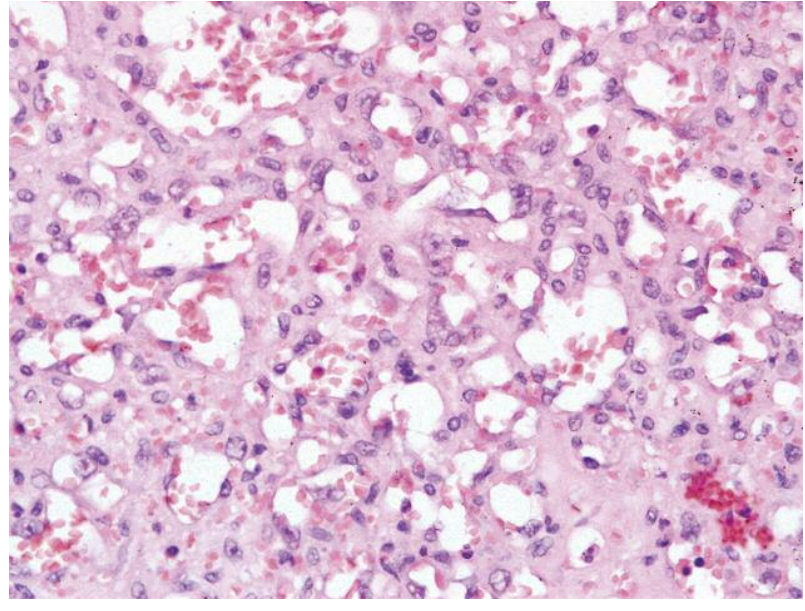

Fig. 6. Angiosarcoma with anastomosing vascular channels. Tumour cells are large, polygonal with abundant eosinophilic cytoplasm and large nuclei with prominent eosinophilic nucleoli

cussed herein died approximately 90 and 30 days after the surgery. Both cases were indeed marked by significant intraperitoneal bleeding, lending further support to the previously produced research. As in the case of the second patient, the co-existence of two independent tumours then coupled with an intraperitoneal haemorrhage appears to have decreased the survival rate even further. Although typically described as rare, primary splenic angiosarcoma nonetheless carries with it an extremely low survival rate upon infection. It has an ever-changing clinical and laboratory profile, with no consideration for genetics, ethnicity, or geographical location [7]. Its diagnostic evasiveness may be further compounded by the fact that although it is typically thought to occur between the $5^{\text {th }}$ and $6^{\text {th }}$ decade of life, it may also strike the paediatric population at virtually any age $[4,7]$. As such, the aforementioned two cases serve to contrast the possible circumstances in which the tumour may occur. The second instance also highlights the first possible reported occurrence of a synchronous presentation of a splenic angiosarcoma alongside a colonic adenocarcinoma, further emphasizing the fact that the former may carry with it a neoplastic risk which extends well beyond distant metastasis.

The authors declare no conflicts of interest.

\section{References}

1. Sordillo EM, Sordill PP, Hajdu SI. Primary hemangiosarcoma of the spleen: report of four cses. Med Pediatr Oncol 1981; 9: 319-24.

2. Hara T, Tsurumi H, Kasahara S. Long-term survival of a patient with splenic angiosarcoma after resection, high-dose chemotherapy, and autologous peripheral blood stem cell transplantation. Intern Med 2010; 49: 2253-7.

3. Falks S, Krishnan J, Meis JM. Primary angiosarcoma of the spleen: a clinicopathologic study of 40 cases. Am J Surg Pathol 1993; 17: 959-70.

4. Neuhauser TS, Derringer GA, Thompson LD, Fanburg-Smith JC, Miettinen M, Saaristo A, Abbondanzo SL. Splenic Angiosarcoma: a cli- 
nopathologic and immunophenotypic study of 28 cases. Mod Pathol 2000; 13: 978-87.

5. Langhans T. Pulsating cavernous neoplasm of the spleen with metastatic nodules to the liver. Virchows Arch Pathol Anat 1879; 75 273-91.

6. Jiménez-Heffernan JA, Hardisson D, Prieto-Nieto MI, Burgos E. Ruptured primary splenic angiosarcoma into the colon: Presentation as anal bleeding. Acta Gastroenterol Belg 1999; 62: 248-51.

7. Manouras A, Giannopolous P, Toufektzian L, et al. Splenic rupture as the presenting manifestation of primary splenic angiosarcoma in a teenage woman: a case report. J Med Case Reports 2008; 2: 133.

8. Aytaç S, Fitoz S, Atasoy C, Kuzu I, Cinar K, Erden I. Mulitmodality demonstration of primary splenic angiosarcoma. J Clin Ultrasound 1999; 27: 92-5.

9. Chung YF, Busmanis I, Hong GS, Soo KC. Splenic angiosarcoma - an unusual cause of bleeding gastrointestinal tract. Singapore J Med 1999; 40: 106-8.

10. Raffel S, Hildebrandt B, Grieser C, Pahl S, Sturm I Thrombocytopenia as first manifestation of splenic angiosarcoma. Ann Hematol 2010; 89: 109-10.

11. Hsu JT, Lin CY, Wu TJ, Chen HM, Hwang TL, Jan YY. Splenic angiosarcoma metastasis to small bowel presented with gastrointestinal bleeding. World J Gastroenterol 2005; 41: 6560-2.

12. Thompson WM, Levy AD, Aguilera NS, Gorospe L, Abbott RM. Angiosarcoma of the spleen: Imaging characteristics in 12 patients. Radiology 2005; 235: 106-15.

\section{Address for correspondence}

\section{Zbigniew Kamocki}

Department of Medical Pathomorphology

Medical University of Bialystok

ul. Waszyngtona 13

15-269 Białystok

e-mail: zkamocki@yahoo.com

Submitted: 26.05 .2011

Accepted: 22.02.2012 